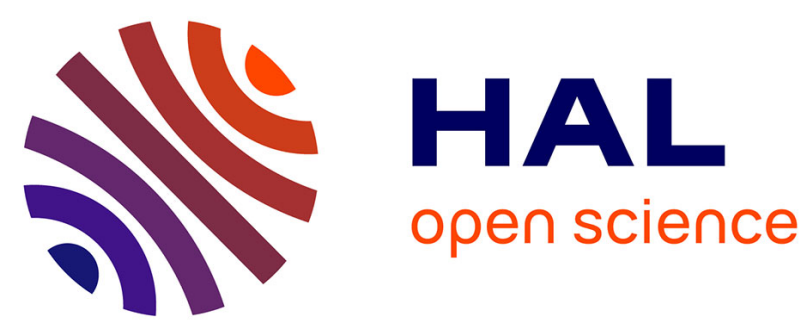

\title{
Zernike ultrasonic tomography for fluid velocity imaging based on pipeline intrusive time-of-flight measurements
}

Nikola Besic, Gabriel Vasile, Andrei Anghel, Teodor-Ion Petrut, Cornel Ioana, Srdjan Stankovic, Alexandre Girard, Guy d'Urso

\section{- To cite this version:}

Nikola Besic, Gabriel Vasile, Andrei Anghel, Teodor-Ion Petrut, Cornel Ioana, et al.. Zernike ultrasonic tomography for fluid velocity imaging based on pipeline intrusive time-of-flight measurements. IEEE Transactions on Ultrasonics, Ferroelectrics and Frequency Control, 2014, 61 (11), pp.1846 - 1855. 10.1109/TUFFC.2014.006515 . hal-01081162

\section{HAL Id: hal-01081162 https://hal.science/hal-01081162}

Submitted on 7 Nov 2014

HAL is a multi-disciplinary open access archive for the deposit and dissemination of scientific research documents, whether they are published or not. The documents may come from teaching and research institutions in France or abroad, or from public or private research centers.
L'archive ouverte pluridisciplinaire HAL, est destinée au dépôt et à la diffusion de documents scientifiques de niveau recherche, publiés ou non, émanant des établissements d'enseignement et de recherche français ou étrangers, des laboratoires publics ou privés. 


\title{
Zernike Ultrasonic Tomography for Fluid Velocity Imaging based on Pipeline Intrusive Time-of-Flight Measurements
}

\author{
Nikola Besic, Student Member IEEE, Gabriel Vasile, Member IEEE, Andrei Anghel, Student Member IEEE, \\ Teodor-Ion Petrut, Student Member IEEE, Cornel Ioana, Member IEEE, Srdjan Stankovic, Senior Member IEEE \\ Alexandre Girard, and Guy d'Urso
}

\begin{abstract}
In this paper we propose a novel Ultrasonic Tomography method for pipeline flow field imaging, based on Zernike polynomial series. Having intrusive multipath time-offlight ultrasonic measurements (difference in flight time and speed of ultrasound) at the input, we provide at the output tomograms of the fluid velocity components (axial, radial and orthoradial velocity). Principally, by representing these velocities as Zernike polynomial series, we reduce the tomography problem to an ill-posed problem of finding the coefficients of the series, relying on the acquired ultrasonic measurements. Thereupon, this problem is treated by applying comparatively the Tikhonov regularization and the Quadratically constrained $\ell_{1}$ minimization. In order to enhance the comparative analysis, we additionally introduce sparsity, by employing the SVD based filtering in selecting Zernike polynomials which are to be included in the series. The first approach - Tikhonov regularization without filtering, imposes as the most suitable one. The performances are quantitatively tested by considering a residual norm and by estimating the flow using the axial velocity tomogram. Finally, the obtained results show the relative residual norm and the error in flow estimation, respectively, $\sim 0.3 \%$ and $\sim 1.6 \%$ for the less turbulent flow and, $\sim 0.5 \%$ and $\sim 1.8 \%$ for the turbulent one. Additionally, a qualitative validation is performed by proximate matching of the derived tomograms with a flow physical model.
\end{abstract}

\section{INTRODUCTION}

Ultrasonic flow metering (UFM) is considered to be one of the most representative non-destructive testing (NDT) measurement techniques for the pipeline flow estimation [1], [2]. Transmission intrusive flowmeters are placed inside the pipeline in order to measure the difference of the transit time of ultrasonic pulses propagating in and against flow direction. Typically, this allows calculating both the speed of sound and the average fluid velocity [3]. The most important constraint is the position of the UFM in the line with respect to discontinuities [4]. However, measuring flow rates inside a pipe flow with the classical time-of-flight method, based on a single ultrasonic propagation path, raises the problem of

Manuscript received Jan. 21, 2014, revised May 16, 2014. and Jul. 2, 2014. N. Besic is with the GIPSA-lab, Grenoble INP, Grenoble, France and with the University of Montenegro, Podgorica, Montenegro (e-mail: nikola.besic@gipsa-lab.grenoble-inp.fr).

G. Vasile, C. Ioana and T. Petrut are with the GIPSA-lab, CNRS/Grenoble INP, Grenoble, France.

A. Anghel is with the GIPSA-lab, Grenoble INP, Grenoble, France and with the University of Politehnica, Bucharest, Romania.

S. Stankovic is with the University of Montenegro, Podgorica, Montenegro.

A. Girard and G. d'Urso are with the R\&D, EDF, Chatou, France. precision and robustness with respect to flow and geometry parameters [5], [6]. Thus, the improvement in measurement accuracy had to be achieved through the integration of several paths on different sections of flow, resulting in far better performances with respect to the estimation of average fluid velocity [7]. As a consequence, the multi-path ultrasonic systems are increasingly appearing as industrial replacement of classical single-path systems [8]. In moving the limits of the range of ultrasonic flowmeters use, RADAR (RAdio Detection And Ranging) tools such as matched filtering are used as well, given that the wide-band signals are taken into consideration [9].

However, the particular interest lies in estimating the flow field in a cross or an oblique section of the pipeline. The mean appearing as most suitable for this purpose is the Ultrasonic Tomography (UT) [10]. Already used in several different domains, UT is ordinarily conceived as a reconstruction method using iterative algebraic methods, spatial Fast Fourier Transform (FFT), Zernike polynomials etc. [11], [12]. In this paper, we propose the Ultrasonic Tomography method for the flow field imaging, based on Zernike polynomials [13].

Zernike polynomials, a set of orthogonal basis functions defined on a continuous unit circle, are firstly introduced by Zernike in his phase contrast method, the improved version of the knife-edge test [14]. In the context of tomography, they are mostly used to describe the wavefront aberrations [15], [16], [17], where they eventually exhibit even better performances than Fourier transform [18].

By representing fluid velocity components as Zernike polynomial series, we derive a method for obtaining corresponding velocity tomograms using intrusive ultrasonic multipath timeof-flight measurements. This approach reduces the ultrasonic tomography to an ill-posed problem, defined by the acquired measurements and the series of appropriate integrated Zernike polynomials. The problem which assumes finding series coefficients, is treated by comparatively using the Tikhonov regularization [19] and the Quadratically constrained $\ell_{1}$ minimization [20]. Under certain constraints, the first method could be treated as $\ell_{2}$ minimization. In order to enhance the comparative analysis by additionally introducing sparsity, we employed, as well, the SVD (Singular Value Decomposition) based filtering in selecting polynomials which have to be integrated in the series.

It appears that the most suitable mean is the Tikhonov reg- 


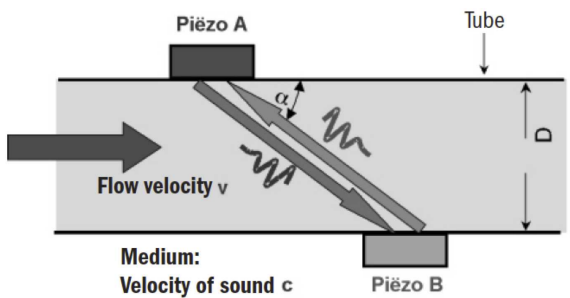

(a)

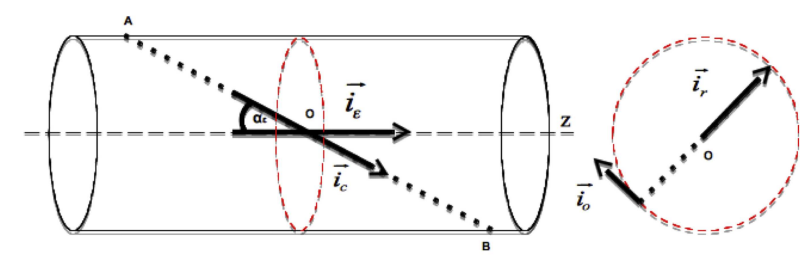

(b)

Fig. 1: Principle of ultrasonic flow measurement (time-of-flight method): (a) schema [23], (b) fluid propagation velocity components.

ularization without filtering. The quantitative decision criteria are the regularization relative residual norm and the comparison of the flow measured by the reference electromagnetic flowmeter with the one estimated using the derived axial tomogram. The obtained results show the relative residual norm and the average error in the flow estimation, respectively, $\sim 0.3 \%$ and $\sim 1.6 \%$ for the less turbulent flow (before the elbow curvature) and, $\sim 0.5 \%$ and $\sim 1.8 \%$ for the turbulent one (after the elbow curvature). Significantly lower residual norm and bigger dynamic range in case of Tikhonov regularization outcome even better flow estimation results obtained by the Quadratically constrained $\ell_{1}$ minimization. Qualitative validation is performed through the comparison with the reference physical model of flow in a pipe with an elbow curvature [21], [22].

The article is organized as follows: in Section II we provide a brief theoretical background by introducing the basic principles of the intrusive ultrasonic time-of-flight flowmeter. Further, section III, the core of the article, describes the proposed method in details. In section IV we present the results followed by the appropriate discussion and finally, we conclude the article with section V.

\section{ULTRASONIC MEASUREMENTS}

This section aims to briefly provide few details concerning basic principles of intrusive ultrasonic time-of-flight measurements.

Short ultrasonic pulses propagating through the liquid flowing in the pipeline, are emitted and received by piezoelectric converters (piëzos) [23], placed in a longitudinal direction on two opposite sides of a pipe with a certain offset (Fig. 1a). The propagation of ultrasonic pulses is influenced by the pipeline flow in the same manner as a canoe crossing diagonally a river: propagation time (time-of-flight) will be shorter when crossing in river flow direction than against it. These two propagation times depend on the velocity of river flow and the velocity of canoe. It is exactly equivalent for the ultrasonic time-of-flight measurements with the fluid velocity and the sound speed. The propagation times are to be calculated as [24]:

$$
t_{A B}=\frac{\|\overrightarrow{A B}\|}{c+\vec{v} \cdot \frac{\overrightarrow{A B}}{\|\overrightarrow{A B}\|}} \approx \frac{1}{c^{2}}(c\|\overrightarrow{A B}\|-\vec{v} \cdot \overrightarrow{A B})
$$

and

$$
t_{B A}=\frac{\|\overrightarrow{B A}\|}{c-\vec{v} \cdot \frac{\overrightarrow{B A}}{\|\overrightarrow{B A}\|}} \approx \frac{1}{c^{2}}(c\|\overrightarrow{B A}\|+\vec{v} \cdot \overrightarrow{B A}),
$$

with $c$ being the average speed of sound and $\vec{v}$ the fluid velocity vector. Given the non-uniform velocity distribution in a pipeline, the difference between times-of-flight can be further expressed as a line integral:

$$
\Delta t=t_{B A}-t_{A B}=2 \int_{C} \frac{\vec{v} \cdot d \vec{s}}{c^{2}},
$$

where $C$ is the domain of integration, representing a straight line defined by vector $\overrightarrow{A B}$. Although it depends on the type of the liquid in the pipeline, the average speed of sound can be found as:

$$
c=\frac{2\|\overrightarrow{A B}\|}{t_{A B}+t_{B A}} .
$$

The velocity of the fluid propagating along the pipeline is expressed as a vector:

$$
\vec{v}=\left(\begin{array}{c}
\overrightarrow{v_{r}} \\
\overrightarrow{v_{o}} \\
\overrightarrow{v_{\varepsilon}}
\end{array}\right)
$$

composed of the radial $\left(v_{r}\right)$, orthoradial $\left(v_{o}\right)$ and axial $\left(v_{\varepsilon}\right)$ component. Therefore, finally, the fluid's flow impacts the ultrasound propagation through the difference in time flight is:

$$
\begin{aligned}
\Delta t & =2 \int_{C} \frac{\vec{v} \cdot d \vec{s}}{c^{2}} \\
& =\frac{2}{c^{2}} \int_{C}\left(\left|\overrightarrow{v_{\varepsilon}}\right| \cos \alpha_{\varepsilon}+\left|\overrightarrow{v_{r}}\right| \cos \alpha_{r}+\left|\overrightarrow{v_{o}}\right| \cos \alpha_{o}\right) d s
\end{aligned}
$$

The derived equation are based on the plane wave assumption, which represents a sort of simplification. The wave propagation in the pipeline flow is generally far more complex [25], [26]. The accuracy of a velocity estimation using time flight measurements, depends on the fluid flow Reynolds number and the mode of the ultrasonic wave, rather than on the radius of the pipeline and the wave frequency [27]. The influence of the pipeline vibrations cannot be neglected, neither [28].

Although the method derivation was motivated by the existing set of intrusive measurements, the application of the method is not by any means restricted to this kind of measurements. 
(a)

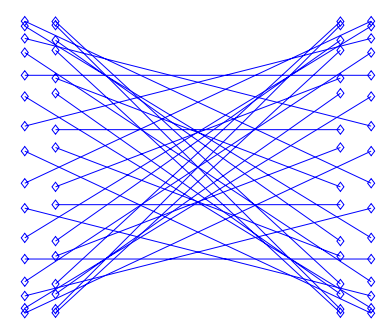

(c)

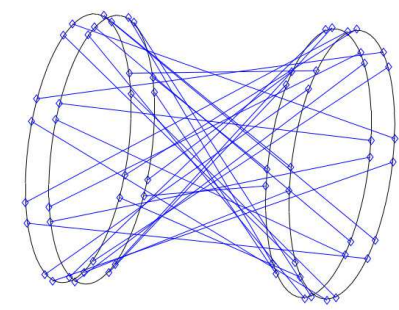

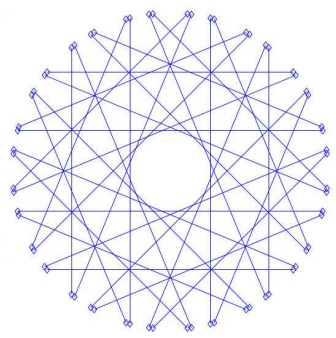

(b)

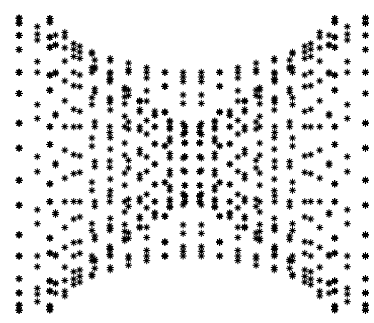

(d)
Fig. 2: The paths of the ultrasound propagation (multiple measurements) in the pipeline: (a) three-dimensional representation, (b) continuous cross section, (c) continuous longitudinal section, (d) discrete longitudinal section.

\section{Ultrasonic TOMOGRAPHY METHOD}

The velocity components in Eq. 6 are functions of distance $s$ (along the line $C$ ). Given the flowmeter configuration (Fig. 1b), if we assume that the velocity distribution doesn't change along the $z$ direction, each $s$ corresponds to a particular point in the pipeline cross-section. Thus, a conversion to a cylindrical coordinate system appears to be suitable. This way, velocities themselves will be defined in a polar coordinate system, which is even intuitive, given the defined purpose deriving the tomogram.

In suitable cylindrical coordinate system, Eq. 6 can be rewritten in the following manner:

$$
\begin{aligned}
\frac{\Delta t_{i} c_{i}^{2}}{2} & =\int_{C}\left|\overrightarrow{v_{\varepsilon}}\right| \cos \alpha_{\varepsilon}^{i} d s+\int_{C}\left|\overrightarrow{v_{r}}\right| \cos \alpha_{r}^{i} d s+ \\
& +\int_{C}\left|\overrightarrow{v_{o}}\right| \cos \alpha_{o}^{i} d s= \\
& =\int_{\theta} \int_{r}\left|\overrightarrow{v_{\varepsilon}}(\theta, r)\right| \cos \alpha_{\varepsilon}^{i} d r d \theta+ \\
& +\int_{\theta} \int_{r}\left|\overrightarrow{v_{r}}(\theta, r)\right| \cos \alpha_{r}^{i} d r d \theta+ \\
& +\int_{\theta} \int_{r}\left|\overrightarrow{v_{o}}(\theta, r)\right| \cos \alpha_{o}^{i} d r d \theta \quad i=1,2, \ldots N .
\end{aligned}
$$

where $N$ is the total number of measurements, or the ultrasound paths. This system of equations, coming from the multipath measurements is the only way to derive the tomogram. Having classical, single-path measurement could lead to the estimation of the average fluid velocity, but not the flow field. This is obvious from the Eq. 6 which can not be, by any means, solved in the context of deriving $\vec{v}_{\varepsilon}(s), \vec{v}_{r}(s)$ and $\vec{v}_{o}(s)$. Even if it was possible, we cannot derive the entire tomogram, but simply velocities corresponding to one diameter of the pipeline cross-section.
As it can be seen in Fig. 2, these multiple paths have to be discretized along $z$ in order to computationally solve the double integrals in Eq. 7. However, this sort of system, in this form, can not be solved neither. Therefore, in our method, in order to be able to derive velocities in polar coordinates (tomograms), we firstly represent each of them as the Zernike polynomial expansion. That way, we reduce the problem to the ill-posed problem of finding expansion coefficients for each of the addends (velocities).

\section{A. Zernike polynomials}

Zernike polynomials represent a set of polynomials defined on a unit circle [29] (Fig. 3). In polar coordinate system, they are given as a product of radial polynomials and angular functions, with the former being developed from the Jacobi polynomials [30], and the latter being the basis functions for the two-dimensional rotation group:

$$
\begin{gathered}
\left.\begin{array}{l}
Z_{j}^{\text {even }}=\sqrt{n+1} R_{n}^{m}(r) \sin m \theta \\
Z_{j}^{\text {odd }}=\sqrt{n+1} R_{n}^{m}(r) \cos m \theta
\end{array}\right\} m \neq 0, \\
\quad Z_{j}=\sqrt{n+1} R_{n}^{0}(r), \quad m=0,
\end{gathered}
$$

where radial polynomial is defined as:

$$
R_{n}^{m}(r)=\sum_{s=0}^{(n-m) / 2} \frac{(-1)^{s}(n-s) !}{s !\left(\frac{n-m}{2}-s\right) !\left(\frac{n+m}{2}-s\right) !} r^{n-2 s} .
$$

The principal advantages of Zernike polynomials, in terms of mathematical properties, are their orthogonality over the continuous unit circle, and the fact that they represent a complete set. Consequently, they can represent arbitrarily complex continuous surfaces, given enough terms [31]. Due to this, they are commonly used in a polynomial expansion of an arbitrary wave front over a circular aperture [30]:

$$
f(\theta, r)=\sum_{j=0}^{n} k_{j} Z_{j}(\theta, r)
$$

with $f(\theta, r)$ being the arbitrary function and $k_{j}$ the expansion coefficient. Therefore, they appear to be a suitable representation of our velocity components. By replacing Eq. 10 in Eq. 7 we are able to express the acquired ultrasound measurements as:

$$
\begin{aligned}
\frac{\Delta t_{i} c_{i}^{2}}{2} & =\sum_{j=0}^{n} a_{j} \int_{\theta} \int_{r} Z_{j}(\theta, r) \cos \alpha_{\varepsilon}^{i} d r d \theta+ \\
& +\sum_{j=0}^{n} b_{j} \int_{\theta} \int_{r} Z_{j}(\theta, r) \cos \alpha_{r}^{i} d r d \theta+ \\
& +\sum_{j=0}^{n} c_{j} \int_{\theta} \int_{r} Z_{j}(\theta, r) \cos \alpha_{o}^{i} d r d \theta
\end{aligned}
$$

That allows us to define the system of equations in the matrix form:

$$
m_{i}=\frac{\Delta t_{i} c_{i}^{2}}{2}=\mathbf{T}_{i}^{a} \mathbf{a}^{\prime}+\mathbf{T}_{i}^{b} \mathbf{b}^{\prime}+\mathbf{T}_{i}^{c} \mathbf{c}^{\prime}, \quad i=1,2, \ldots N
$$




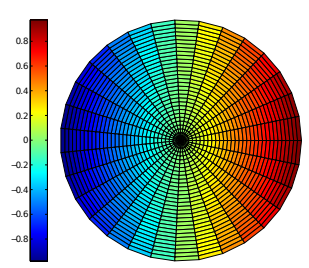

(a)

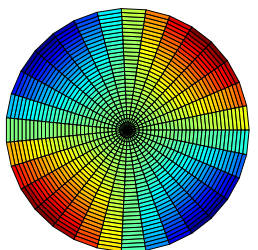

(b)

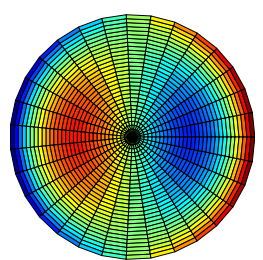

(c)

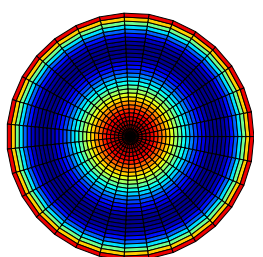

(d)

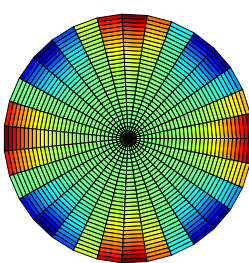

(e)

Fig. 3: Zernike polynomials $\left(Z_{j}\right)$ : (a) $Z_{2}$, (b) $Z_{5}$, (c) $Z_{8}$, (d) $Z_{11}$, (e) $Z_{14}$.

where $\mathbf{a}, \mathbf{b}$ and $\mathbf{c}$ are the vectors containing the unknown coefficients and $\mathbf{T}_{i}^{a}, \mathbf{T}_{i}^{b}$ and $\mathbf{T}_{i}^{c}$ the rows of the transformation matrices i.e. solutions of the previously defined integrals for each $j$, where $i$ is the index of measurement ( $N$ in total). $\mathbf{m}$ is the vector of measurements. The discrete ill-posed problem is finally defined as:

$$
\left[\begin{array}{lll}
\mathbf{T}^{a} & \mathbf{T}^{b} & \mathbf{T}^{c}
\end{array}\right]\left[\begin{array}{l}
\mathbf{a}^{\prime} \\
\mathbf{b}^{\prime} \\
\mathbf{c}^{\prime}
\end{array}\right]=\mathbf{T x}=\mathbf{m} .
$$

\section{B. Tikhonov Regularization}

In order to be able to reconstruct velocity distribution, we ought to find coefficients $\mathbf{x}$ by solving discrete ill-posed problem [19] designated in Eq. 13. The useful and stable solution of this problem is obtained through the regularization [32]. Certainly, the most common form of the regularization and therefore the first applied method, is Tikhonov regularization [33], [34], where the regularized solution $\mathbf{x}_{\lambda}$ is defined as a minimizer of the following weighted combination of the residual norm and the side constraint:

$$
\mathbf{x}_{\lambda}=\operatorname{argmin}\left(\|\mathbf{T} \mathbf{x}-\mathbf{m}\|_{2}^{2}+\lambda^{2}\left\|\mathbf{L}\left(\mathbf{x}-\mathbf{x}_{\mathbf{0}}\right)\right\|_{2}^{2}\right),
$$

with $\lambda$ being the regularization parameter, $\mathbf{L}$ the identity matrix and $\mathbf{x}_{\mathbf{0}}$ the vector of initial conditions. The regularization parameter has significant impact on solution properties, through controlling the weight of minimization of the side constraint and the sensitivity of the solution with respect to perturbations in $\mathbf{T}$ and $\mathbf{m}$.

As it appears, the most utilized algorithm for the stated least square problem (Eq. 14) is the one based on QR factorisation [35]. However, we rather rely on the MATLAB toolbox proposed by Hansen [36], [37], who chooses another approach, based on Singular Value Decomposition (SVD) of the matrix T:

$$
\mathbf{T}=\mathbf{U} \boldsymbol{\Sigma} \mathbf{V}^{T}=\sum_{i=1}^{n} \mathbf{u}_{i} \sigma_{i} \mathbf{v}_{i}^{T}
$$

By assuming $\mathbf{L}=I_{N}$ the regularized solution is given as:

$$
\mathbf{x}^{r e g}=\sum_{i=1}^{n} f_{i} \frac{\mathbf{u}_{i}^{T} \mathbf{m}}{\sigma_{i}} \mathbf{v}_{i},
$$

where $f_{i}$ are filter factors assuring that the addends corresponding to the smaller singular values are filtered out. In case of a Tikhonov regularization, they are defined as:

$$
f_{i}=\frac{\sigma_{i}^{2}}{\sigma_{i}^{2}+\lambda^{2}} .
$$

The regularization parameter $\lambda$ is determined using Generalized Cross Validation (GCV) [38], by minimizing the GCV function:

$$
G=\frac{\left\|\mathbf{T} \mathbf{x}^{r e g}-\mathbf{m}\right\|_{2}^{2}}{\left(\operatorname{trace}\left(\mathbf{I}_{N}-\mathbf{T} \mathbf{T}^{\prime}\right)\right)^{2}} .
$$

\section{Quadratically constrained $\ell_{1}$ minimization}

The alternative approach assumes using the quadratically constrained $\ell_{1}$ minimization [20], [39] in deriving unknown Zernike coefficients. In this case, we search minimal value of $\mathrm{x}$ satisfying the inequality:

$$
\frac{1}{2}\left(\|\mathbf{T} \mathbf{x}-\mathbf{m}\|_{2}^{2}-\epsilon^{2}\right) \leq 0 .
$$

The problem is reformulated as the second order cone problem [40]:

$$
\begin{aligned}
\min _{\mathbf{x}, \mathbf{u}} \sum_{i} u_{i} \quad \text { subject to } \mathbf{x}-\mathbf{u} & \leq 0, \\
-\mathbf{x}-\mathbf{u} & \leq 0 \\
\frac{1}{2}\left(\|\mathbf{T} \mathbf{x}-\mathbf{m}\|_{2}^{2}-\epsilon^{2}\right) & \leq 0,
\end{aligned}
$$

where the first two inequalities assure that $\mathbf{u}$ is positive, therefore that the cost-function is non-negative. The problem is solved using the log-barrier method [41].

\section{SVD based filtering of Zernike polynomials}

The filtering is applied in deriving matrices $\mathbf{T}$. The contribution of Zernike polynomial to the integration is estimated by applying the Singular Value Decomposition (SVD) to the matrix $\mathbf{T}$, after adding a new polynomial to the expansion (new column). As the criterion we take a minimal singular value, which is compared with the predefined threshold $(t=0.001)$. This way, we obtain sparse Zernike representation of the velocity.

This preprocessing is combined both with the Tikhonov Regularization and the Quadratically constrained $\ell_{1}$ minimization. However, given that the former can be equalized with $\ell_{2}$ minimization, in this case imposing "more strict" sparsity of the Zernike representation should influence the results more than in case of $\ell_{1}$ minimization. 


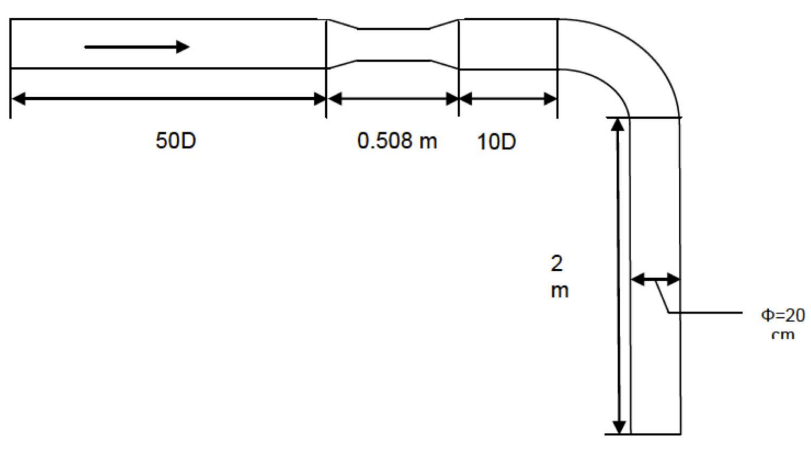

(a)

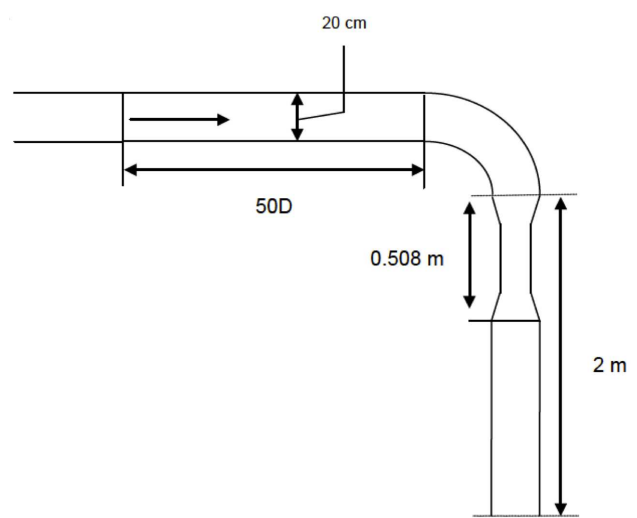

(b)

Fig. 4: Measurements configuration $(\delta=1.5)$ : (a) before the knee, (b) after the knee.

\section{E. Velocity distribution reconstruction}

Finally, knowing the expansion coefficients, velocity tomograms are calculated using Zernike polynomial expansion:

$$
\begin{aligned}
\left|\overrightarrow{\overrightarrow{v_{\varepsilon}}(\theta, r)}\right| & =\sum_{j=0}^{n} \mathbf{a}_{j}^{r e g} Z_{j}(\theta, r), \\
\left|\overrightarrow{\overrightarrow{v_{r}}(\theta, r)}\right| & =\sum_{j=0}^{n} \mathbf{b}_{j}^{r e g} Z_{j}(\theta, r), \\
\left|\overrightarrow{v_{o}(\theta, r)}\right| & =\sum_{j=0}^{n} \mathbf{c}_{j}^{r e g} Z_{j}(\theta, r) .
\end{aligned}
$$

The results we present in the following section are based on the polynomials expansion up to the order $n=13$. This choice is based on the empirical assertion that, in the considered case, the inclusion of higher orders does not influence the obtained tomograms.

\section{RESULTS}

We applied the proposed method to the measurements acquired using DFX MM US flowmeter [42] in a pipeline with the radius $R \approx 100 \mathrm{~mm}$ over a distance $(z)$ of $D \approx 508 \mathrm{~mm}$. Radius of curvature to pipeline diameter ratio is $\delta=1.5$. Multipath measurements assume in this case $N=32$ different paths. Each of the paths is discretized in 1024 points.

The first set of measurements was acquired before the elbow, where the propagating fluid can be considered as a turbulent fully-developed flow, while the second set, taken after the elbow, represent a turbulent disturbed flow (Fig. 4).

The quality of the proposed imaging method was analysed with respect to two criteria:

- residual norm: indicator of the suitability of the employed regularization/minimization method:

$$
R=\frac{\|\mathbf{T} \mathbf{x}-\mathbf{m}\|_{2}^{2}}{\|\mathbf{m}\|_{2}^{2}} .
$$

- flow: the volumetric flow rate, computed using derived axial component:

$$
Q=\int_{\theta} \int_{r}\left|\widehat{\overrightarrow{v_{\varepsilon}}(\theta, r)}\right| r d r d \theta .
$$

In Table I, we provide the quantitative results achieved with four tested approaches: Tikhnov regularization and Quadratically constrained $\ell_{1}$ minimization, with and without filtering of polynomials. The obtained velocity tomograms are presented in Fig. 5 and 6.

The residual norm reflects the capability of the employed regularization/minimization method to face the illpossessedness of the problem. This parameter implies the error in the Zernike polynomial expansion coefficients' estimation. It can be considered as the error in the reconstruction of timeof-flight measurements, using derived tomograms.

The flow relative error ("flow error") is calculated by comparing the estimated flow (Eq. 23) to the one measured using reference electromagnetic flowmeter [43].

As a mean of qualitative validation the obtained tomograms are compared with the reference flow physical model (Fig. 7), as well [43]. This validation is reinforced through the additional comparison with the computational fluid dynamics (CFD) calculation for the pipeline characterised with the same $\delta$, provided in Fig. 18 in [22].

On one side, the relative residual norm indicates the superiority of the Tikhonov regularization method in facing the ill-possessedness of the problem. On the other side, the flow relative error shows that the more accurate estimation is achieved with Quadratically constrained $\ell_{1}$ minimization.

Employed filtering does not improve the performances for none of two approaches, with respect to both of the defined criteria. It almost does not affect the flow estimation while degrading the relative residual norm in the former case. In the latter one, the results are virtually identical, which was the reason for not illustrating them in Fig. 5 and 6.

However, significantly bigger dynamic range in case of the Tikhonov regularization (Fig. 5 and 6), closer to the one expected by a simulated flow physical model, imposes this approach as the most appropriate choice for the proposed method.

Very low magnitudes of the estimated radial and orthoradial velocity tomograms lead to the eventual ambiguity with the 

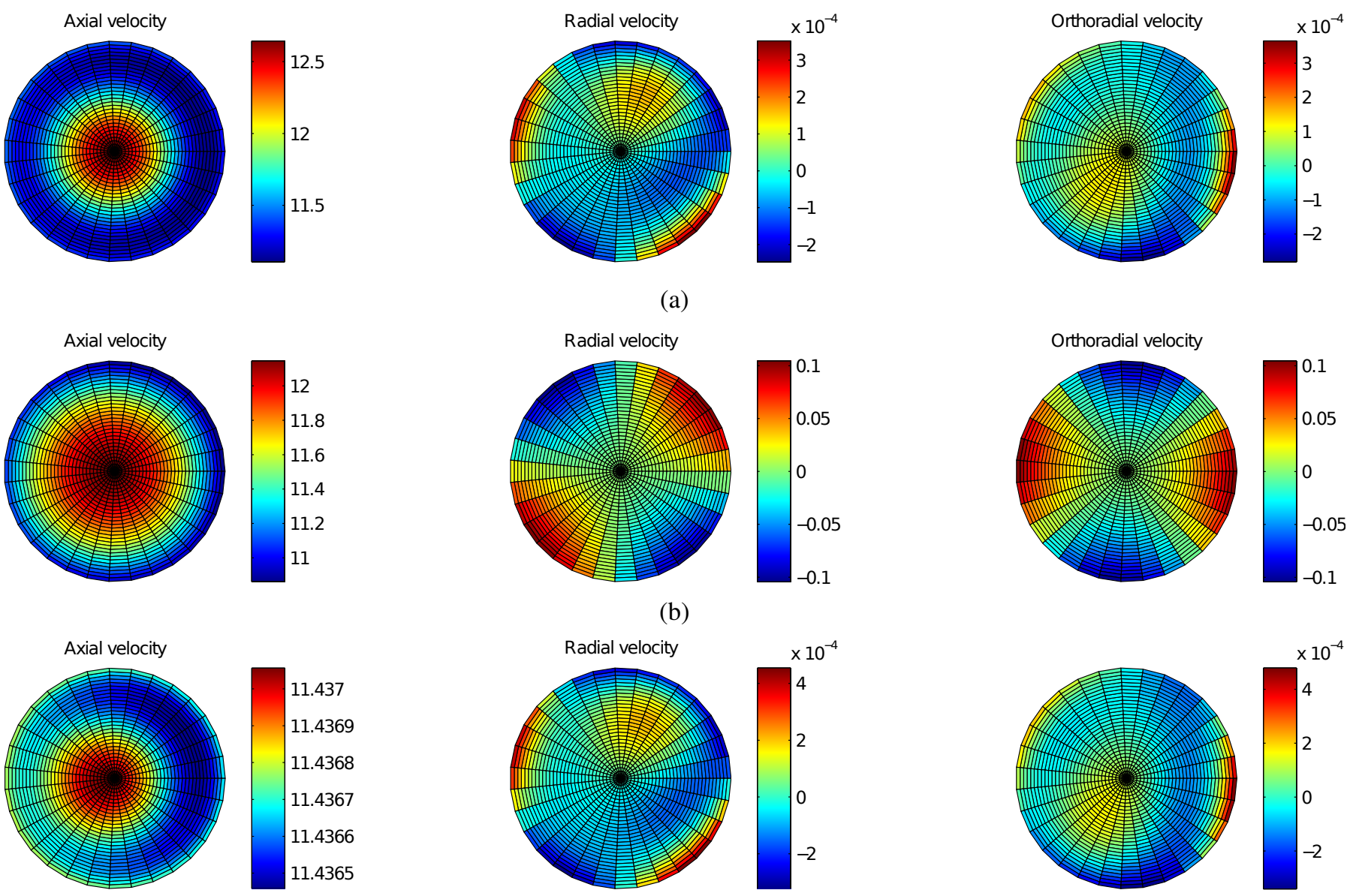

Fig. 5: Tomograms of the dimensionless velocity for a fully-developed flow (before the elbow): (a) Tikhonov regularization without filtering, (b) Tikhonov regularization with filtering, (c) Quadratically constrained $\ell_{1}$ minimization without filtering.

noise (Fig. 5 and 6), leaving the axial velocity tomogram as the most relevant output in the proposed method.

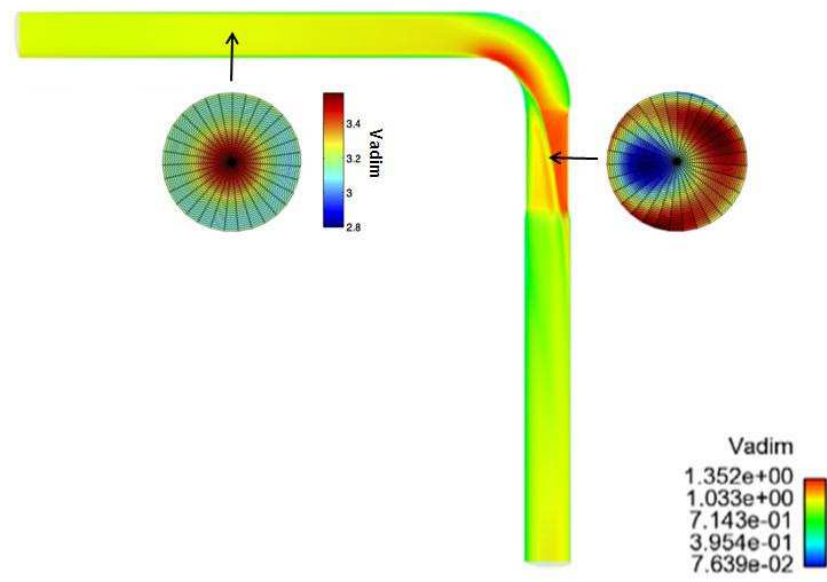

Fig. 7: The comparison of the obtained tomograms with the longitudinal section of the flow physical model $(\delta=1.5)[43]\left(Q=300 \mathrm{~m}^{3} / h\right)$.

The presented quantitative validation demonstrate the plausibility of the proposed method in terms of capability to represent the acquired measurements using ultrasonic tomo- grams. Furthermore, the comparison with the simulated flow physical model, qualitatively upholds the fidelity of the derived tomograms.

\section{CONClusion}

This paper presents a method allowing the reconstruction of a transverse flow field in a pipeline, using intrusive timeof-flight ultrasonic measurements. Given the outputs, having a form of velocities images, it genuinely represents one Ultrasonic Tomography (UT) method. By introducing Zernike polynomial expansions instead of velocity components, we reduced the UT problem to one ill-posed problem of finding the coefficients of the expansion. Further, we have applied comparatively two approaches in treating this problem: Tikhonov regularization and Quadratically constrained $\ell_{1}$ minimization. Both of the approaches were eventually reinforced with the SVD filtering of Zernike polynomials, as well. However, the former one, without filtering, proved to be the most suitable with respect to the performed validation, which assumed both the relative residual norm and the flow estimation using obtained tomograms. Finally, the obtained results in terms of the average relative error indicate for a fully-developed flow $\sim 0.3 \%$ and $\sim 1.6 \%$ in residual norm and flow estimation respectively, and $\sim 0.5 \%$ and $\sim 1.8 \%$ for a disturbed one. 

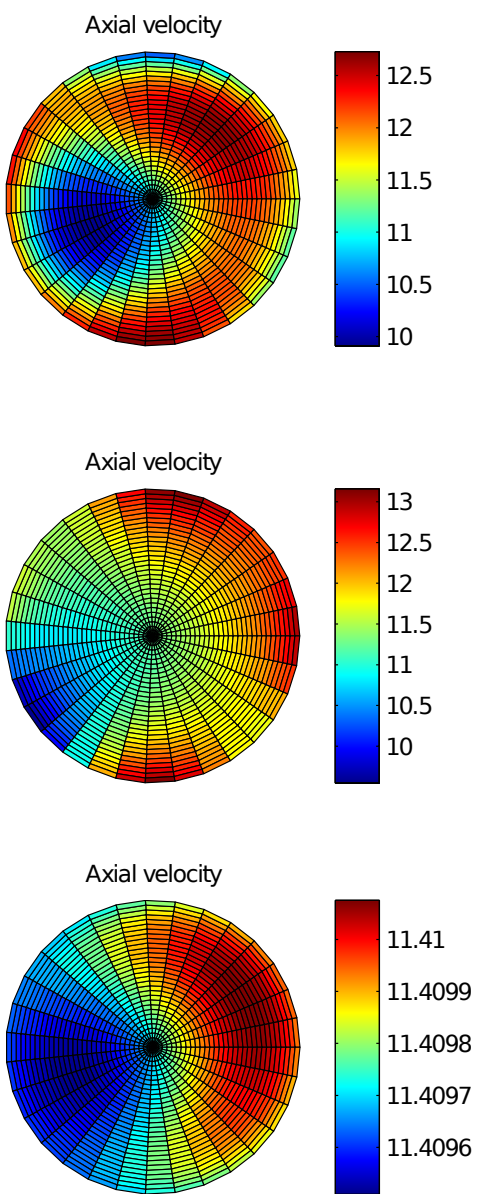

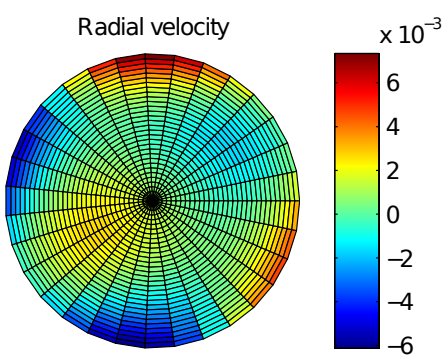

(a)

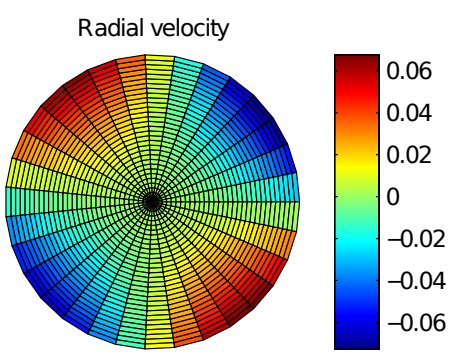

(b)

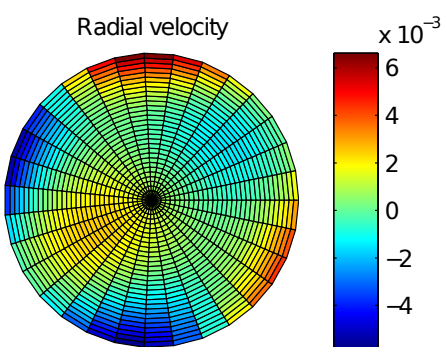

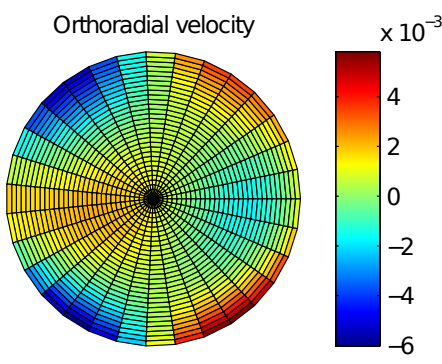
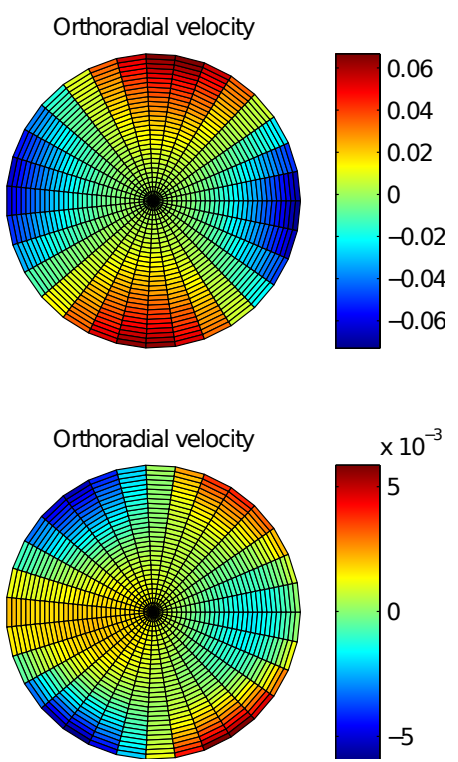

(c)

Fig. 6: Tomograms of the dimensionless velocity for a disturbed flow (after the elbow): (a) Tikhonov regularization without filtering, (b) Tikhonov regularization with filtering, (c) Quadratically constrained $\ell_{1}$ minimization without filtering.

\section{ACKNOWLEDGEMENTS}

This work has been done as a part of the collaboration with the Électricité de France (EDF). In part, it has been supported, by the "Smart Hydro Monitoring" Tenerrdis project. We ought to thank to $R \& D$ department of the $E D F$ for the provided measurements. We owe a particular gratitude to anonymous reviewers for their suggestions and advices, which improved significantly the quality of the presented work.

\section{REFERENCES}

[1] G. T. Herman, Nondestructive Testing. Materials Park, Ohio, USA: ASM International, 1995.

[2] L. Lynnworth and Y. Liu, "Ultrasonics flowmeters: Half-century progress report, 1955-2005," Ultrasonics, vol. 44, no. 1, pp. 1371-1378, 2006.

[3] L. Lynnworth, Ultrasonic Measurements for Process Control. San Diego, USA: Academic Press, 1989.

[4] T. Strunz, A. Wiest, A. Fleury, and T. Frohlich, "Influence of turbulence on ultrasonic flow measurements," in 5th IGHEM Conference, Lucerne,CH, 2004.

[5] J. Reyes and A. Acevedo, "Modeling and simulation of ultrasonic flow meters: State of art," in ANDESCON, 2010 IEEE, 2010, pp. 1-7.

[6] J. C. Jung and P. H. Seong, "Estimation of the flow profile correction factor of a transit-time ultrasonic flow meter for the feedwater flow measurement in a nuclear power plant," IEEE Transactions on Nuclear Science, vol. 52, no. 3, 2005.

[7] T. Cousins and D. Augenstein, "Proving of multi-path liquid ultrasonic flowmeters," 20th NSFMW, 2002.
[8] Y. Wang, "Development of multi-path ultrasonic flow meter based on embedded system," in Control and Automation (ICCA), 2010 8th IEEE International Conference on, 2010, pp. 689-692.

[9] T. Folkestad and K. S. Mylvaganam, "Chirp excitation of ultrasonic probes and algorithm for filtering transit times in high-rangeability gas flow metering," IEEE Transactions on Ultrasonics, Ferroelectrics, and Frequency Control, vol. 40, no. 3, pp. 193-215, May 1993.

[10] J. W. Eberhard, "Ultrasonic tomography for nondestructive evaluation," Annual Review of Materials Science, vol. 12, pp. 1-21, 1982.

[11] P. Lasaygues, R. Guillermin, and J.-P. Lefebvre, Bone Quantitative Ultrasound. Springer Netherlands, 2010, ch. Ultrasonic Computed Tomography, pp. 441-459.

[12] D. Kurniadi and A. Trisnobubi, "A multi-path ultrasonic time flow meter using a tomography method for gas flow velocity profile measurement," Particle and Particle Systems Characterization, vol. 23, no. 3-4, pp. 330-338, 2006.

[13] G. T. Herman, Image Reconstructions from Projections. New York, NY, USA: Academic Press, 1980.

[14] F. Zernike, "Diffraction theory of knife-edge test and its improved form, the phase contrast method," Monthly Notices of the Royal Astronomical Society, vol. 94, pp. 377-384, 1934.

[15] G. N. Lawrence and W. W. Chow, "Wave-front tomography by zernike polynomial decomposition," Optics Letters, vol. 9, pp. 267-269, 1984.

[16] V. N. Mahajan, Zernike polynomials and wavefront fitting. San Diego, USA: Academic Press, 1989.

[17] — Optical Shop Testing. New York, USA: Wiley, 2007, ch. Zernike polynomials and wavefront fitting.

[18] G. Yoon, S. Pantanelli, and S. MacRae., "Comparison of zernike and fourier wavefront reconstruction algorithms in representing corneal aberration of normal and abnormal eyes," J. Refract. Surg., vol. 24, no. 6 , pp. 582-590, 2008. 
TABLE I: COMPARISON OF DIFFERENT METHODS IN TERMS OF RELATIVE RESIDUAL NORM AND FLOW ERROR.

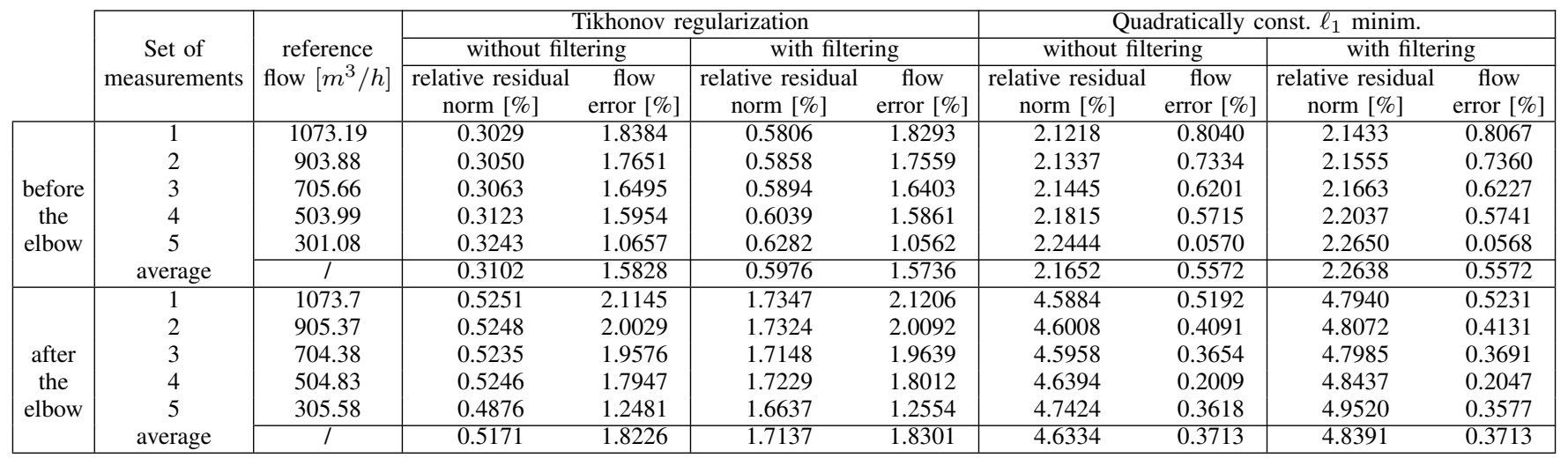

[19] J. Hadamard, Lectures on Cauchy's Problem in Linear Partial Differential Equa-tions. New Haven, USA: Yale University Press, 1923.

[20] E. J. Candes, J. Romberg, and T. Tao, "Stable signal recovery from incomplete and inaccurate measurements," Comm. Pure Appl. Math., vol. 59, no. 8, pp. 1207-1223, 2006.

[21] A. Ono, N. Kimura, H. Kamide, and A. Tobita, Influence of elbow curvature on flow structure at elbow outlet under high Reynolds number condition. Oarai Research and Development Center, 2010.

[22] S. Hambric, D. Boger, J. Fahnline, and R. Campbell, "Structure and fluidborne acoustic power sources induced by turbulent flow in $90^{\circ}$ piping elbows," Journal of Fluids and Structures, vol. 26, pp. 121-147, 2010.

[23] F. Hofmann, Fundamentals of Ultrasonic flow measurement for industrial applications. Duisburg, DE: KROHNE Messtechnik GmbH and Co. KG, 2000.

[24] E. Mandard, D. Kouamé, R. Battault, J.-P. Remenieras, and F. Patat, "Methodology for developing a high-precision ultrasound flow meter and fluid velocity profile reconstruction," IEEE Transactions on Ultrasonics, Ferroelectrics, and Frequency Control, vol. 55, no. 1, pp. 161-172, 2008.

[25] Y. Chen, Y. Huang, and X. Chen, "Ultrasonic wave propagation in thermoviscous moving fluid confined by heating pipeline and flow measurement performance," J. Acoust. Soc. Am., vol. 134, pp. 1863$1874,2013$.

[26] _ , "Viscothermal longitudinal wave propagation in non-uniform shear liquid flow confined by constant temperature pipeline and implications for ultrasonic flow meter," Acta Acust united Ac., vol. 99, pp. 875-890, 2013.

[27] M. Willatzen, "Liquid flows and vibration characteristics of straight-tube cylindrical shells," J. Sound Vib., vol. 260, no. 3, pp. 417-429, 2003.

[28] — , "Sound propagation in a moving fluid confined by cylindrical walls-a comparison between an exact analysis and the local-plane wave approximation," J. Sound Vib., vol. 247, no. 3, pp. 719-729, 2001.

[29] R. J. Noll, "Zernike polynomials and atmospheric turbulence," Journal of Optical Society of America, vol. 66, no. 3, pp. 207-211, 1976.

[30] M. Born and E. Wolf, Principles of Optics: Electromagnetic theory of propagation, interference and diffraction of light. Oxford, UK: Pergamon Press, 1965.

[31] C. H. Teh and R. T. Chin, "On the image analysis by the methods of moments," IEEE Trans. Pattern Anal. Mach. Intell., vol. 10, no. 4, pp. 496-512, 1988.

[32] M. Bertero, C. D. Mol, and E. R. Pike, "Linear inverse problems with discrete data: Ii. stability and regularization," Inverse Problems, vol. 4, pp. 573-594, 1988

[33] A. N. Tikhonov, "Solution of incorrectly formulated problems and the regulariza- tion method," Dokl. Akad. Nauk. SSSR, vol. 151, pp. 501504, 1963.

[34] A. N. Tikhonov and V. Y. Arsenin, Solutions of Ill-Posed Problems. Washington, D.C. ,USA: Winston and Sons, 1977.

[35] L. Elden, "Algorithms for regularization of ill-conditioned least-squares problems," BIT, vol. 17, pp. 134-145, 1977.

[36] P. C. Hansen, "Regularization tools: A matlab package for analysis and solution of discrete ill-posed problems," Numerical Algorithms, vol. 6, pp. 1-35, 1994

[37] — , "Regularization tools version 4.0 for matlab 7.3," Numerical Algorithms, vol. 46, pp. 189-194, 2007.
[38] G. Wahba, Spline Models for Observational Data. Philadelphia, USA: SIAM, 1990

[39] R. Tibshirani, "Regression shrinkage and selection via the lasso," $J$. Royal. Statist. Soc B., vol. 58, no. 1, pp. 267-288, 1996.

[40] E. Candès and J. Romberg, "L1-magic: Recovery of sparse signals via convex programming," October 2005, http://www.acm.caltech.edu/11magic.

[41] S. Boyd and L. Vandenberghe, Convex Optimization. Cambridge, UK: Cambridge University Press, 2004

[42] D. Laurent, "Technical training documents," Metering \& Technology SAS (M\&T), Tech. Rep., 2010.

[43] J. Veau, A. Girard, and M. Y. Chaouche, "Mesure du profil de vitesse à l'aide d'un débitmètre ultrasonore 32 cordes," EDF R\&D, Tech. Rep., March 2013, (in French).

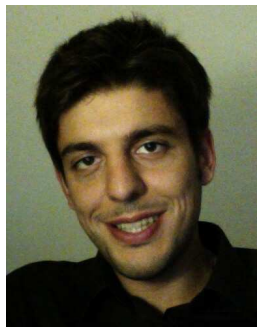

Nikola Besic (S'10) was born in Podgorica, Montenegro, in November 1987. He received the B.Sc. degree in electrical engineering in 2009 from the University of Montenegro and the M.Sc. degree in optics and RF engineering in 2011 from the Grenoble Institute of Technology (Phelma/Grenoble INP). Currently, as a French Government fellow, with the Grenoble-Image-sPeech-Signal-Automatics Laboratory (GIPSA-lab) in Grenoble, he is jointly pursuing the Doctorate degree in signal and image processing, from the University of Grenoble, and the $\mathrm{Ph} . \mathrm{D}$. degree in technical sciences, from the University of Montenegro.

His current research is mostly related to radar and optical remote sensing, with a particular emphasis on the radar polarimetry and the remote sensing of cryosphere. His research interests also include other signal and image processing techniques, tightly bound to physics of electromagnetic and mechanical waves.

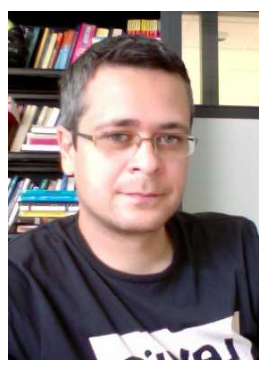

Gabriel Vasile (S'06-M'07) received the M.Eng. degree in electrical engineering and computer science and the M.S. degree in image, shapes, and artificial intelligence from the POLITEHNICA University, Bucharest, Romania, in 2003 and 2004, respectively, and the Ph.D. degree in signal and image processing from the Savoie University, Annecy, France, in 2007. From 2007 to 2008, he was a Postdoctoral Fellow with the French Space Agency (CNES) and was with the French Aerospace Laboratory (ONERA), Palaiseau, France. In 2008, he joined the French National Council for Scientific Research (CNRS), where he is currently a Research Scientist and a member of the Grenoble Image Speech Signal Automatics Laboratory, Grenoble, France.

$\mathrm{He}$ is author of more than 100 papers published in international journals and conference proceedings. In 2008, he received the "Bourse de la Vocation Prix des Neiges" to recognize his work in radar remote sensing for the benefit of the Savoie and the Haute Savoie counties, France.

His current research interests include signal and image processing, radar and acoustics remote sensing, polarimetry, interferometry, and tomography. 


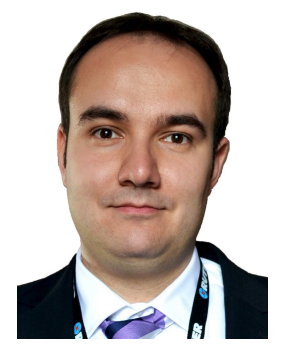

Andrei Anghel (S'11) received the Engineers degree (as valedictorian) and the Masters degree (with the highest grade) in electronic engineering and telecommunications from the University POLITEHNICA of Bucharest, Bucharest, Romania, in 2010 and 2012, respectively.

$\mathrm{He}$ is currently working for a joint Ph.D. degree in the field of radar signal processing from the Grenoble Institute of Technology, Grenoble, France and the University POLITEHNICA of Bucharest, Bucharest, Romania. Since 2012 he is Teaching Assistant at the Faculty of Electronics, Telecommunications and Information Technology within the University POLITEHNICA of Bucharest, Bucharest, Romania. Between 2010-2011 he has worked in the field of metamaterial composite right/left-handed (CRLH) antennas. In 2012 he pursued a research internship at the Grenoble Image sPeech Signal Automatics Laboratory (GIPSA-Lab), Grenoble, France on ground-based FMCW radar signal processing. His current research interests include microwaves, radar and signal processing with applications in infrastructure monitoring.

Mr. Anghel regularly acts as a Reviewer for the IET Electronics Letters and the Progress in Electromagnetics Research (PIER) Journals. He received two gold medals (in 2005 and 2006) at the International Physics Olympiads.

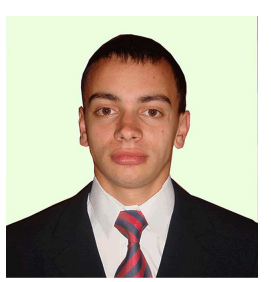

Teodor-Ion Petrut (M12) received the B.S. degree in mechanical engineering from the Military Technical Academy, Bucharest, Romania, in 2010 and the M.S. degree in mechanical engineering from the University Politehnica, Bucharest, Romania, in 2012.

From 2010 to 2012, he was a R\&D engineer with the Military Technical Academy, Bucharest, Romania. From 2012 to 2013 he attended numerous internships with the Gipsa-lab Laboratory, Grenoble Institute of Technology, France, and with the EDF R\&D Chatou, France, on Non-Destructive Testing research studies. He is currently R\&D engineer with the Gipsa-lab where his research interest is mainly focusing on Non-Destructive Evaluation with ultrasounds in the field of hydraulic monitoring and measurement.

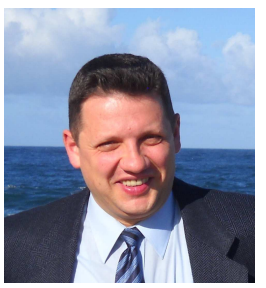

Cornel Ioana received the Dipl.-Eng. degree in electrical engineering from the Romanian Military Technical Academy of Bucharest, Bucharest, Romania, in 1999 and the M.S. degree in telecommunication science and the Ph.D. degree in the electrical engineering field, both from University of Brest, Brest, France, in 2001 and 2003, respectively.

Between 1999 and 2001, he was active as a Military Researcher in a research institute of the Romanian Ministry of Defense (METRA), Bucharest, Romania). Between 2003 and 2006, he worked as Researcher and Development Engineer in ENSIETA, Brest, France. Since 2006, he is Associate Professor-Researcher with the Grenoble Institute of Technology/GIPSA-lab, Grenoble, France. His current research activity deals with the signal processing methods adapted to the natural phenomena. His scientific interests are nonstationary signal processing, natural process characterization, underwater systems, electronic warfare, and real-time systems.

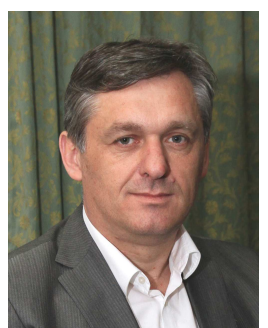

Srdjan Stankovic was born 1964 in Montenegro. He received the B.S. (Hons.) degree from the University of Montenegro, in 1988, the M.S. degree from the University of Zagreb, Croatia, in 1991, and the Ph.D. degree from the University of Montenegro in 1993, all in Electrical Engineering.

From 1988 to 1992 , he worked in the Aluminum Plant of Podgorica as a Research Assistant. In 1992 he joined the Faculty of Electrical Engineering, University of Montenegro, where he is currently a Full Professor. In the period 2007-2013 he served as Dean of the Faculty of Electrical Engineering, University of Montenegro. His interests are in signal processing, multimedia systems, and digital electronics. $\mathrm{He}$ is a member of the Board of Directors in Montenegrin Broadcasting Company since 2004. He was the President of the Board of Directors in Montenegrin Broadcasting Company (2005-2006). In 1998 he spent a period of time with the Department of Informatics at the Aristotle University in Thessaloniki, supported by Greek IKY foundation. In the 1999-2000, he was on leave at the Darmstadt University of Technology, with the Signal Theory Group, supported by the Alexander von Humboldt Foundation. In 2002, he spent three months at the Department of Computer Science, the University of Applied Sciences Bonn-Rhein-Sieg, as an Alexander von Humboldt Fellow. From 2004 to 2006, he stayed several times with the E3I2 Laboratory, ENSIETA, Brest, France. From 2007 to 2010 he visited (one month research stay) Centre for digital signal processing research at King's College London, Laboratory of mathematical methods in image processing, at Moscow State University Lomonosov, as well as GIPSA Laboratory at INPG Grenoble. He spent academic 2012/2013 with the Center for Advanced Communications at the Villanova University, PA.

He has published a book "Multimedia Signals and Systems" by Springer and several textbooks on electronics devices (in Montenegrin). He published about 80 journal papers. In 2010, he was the Lead Guest Editor of the EURASIP Journal on Advances in Signal Processing for the special issue: Time-frequency analysis and its applications to multimedia signals, as well as the Guest Editor of the Signal Processing for special issue: Fourier related transforms. He was the Lead Guest Editor of the IET Signal Processing for the Special issue: Compressive Sensing and Robust Transforms, published in 2014.

From 2005 to 2009 Dr. Stankovic was serving as an Associate Editor of the IEEE Transactions on Image Processing. He is a Senior member of the IEEE. In 2011 he was awarded by the Ministry of Science in Montenegro as the Leader of the Best Scientific Project in Montenegro.

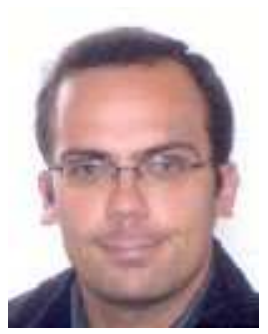

Alexandre Girard received the Engineering degree from the Ecole Centrale de Paris, Paris, France, in 2000 with a specialization in applied mathematics.

He worked in optimization and robust control for power plants from 2000 to 2005 as a consultant. $\mathrm{He}$ is currently a Research Engineer at Electricité de France (EDF) in control and signal processing.

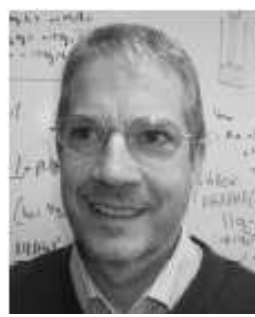

Guy d'Urso received the Engineering degree from ENSIEG, Grenoble, France, in 1993 with a specialization in signal processing.

$\mathrm{He}$ is currently a Research Engineer at Electricité de France (EDF) in signal and image processing, with an expert status. He is also interested in measurement domain. 